Динамика, прочность машин, приборов и аппаратуры

DOI: https://doi.org/10.24866/2227-6858/2021-3-3

УДК 629.4.015;62-752;681.5

А.В. Елисеев, Н.К. Кузнецов, С.В. Елисеев

ЕЛИСЕЕВ АНДРЕЙ ВЛАДИМИРОВИЧ - к.Т.Н., доцент (авТор, ответственный за переписку), SPIN: 8781-1123, ResearcherID: N-9357-2016, ORCID: 0000-0003-0222-2507, ScopusID: 57191957568, eavsh@ya.ru КУЗНЕЦОВ НИКОЛАЙ КОНСТАНТИНОВИЧ - д.Т.Н., профрессор, заведующий кафедрой конструирования и стандартизации в машиностроении, SPIN: 4875-9445, ORCID: 0000-0002-3083-0182, knik@istu.edu Иркутский национальный исследовательский технический университет ЕЛИСЕЕВ СЕРГЕЙ ВИКТОРОВИЧ - Д.Т.Н. профессор, советник при ректорате по научной работе, SPIN: 2792-7608, ORCID: 0000-0001-6876-8786, eliseev_s@inbox.ru Иркутский государственный университет путей сообщения Иркутск, Россия

\title{
Системный анализ в оценке динамических состояний технических объектов: динамическоегашение колебаний, связность внешних воздействий
}

\begin{abstract}
Аннотация: Работа посвящена развитию научно-методических основ современной динамики машин в рамках подходов, связанных с системным анализом и методами структурного математического моделирования. В настоящей статье авторы представляют разработку математического аппарата, позволяющего охарактеризовать разнообразие особенностей динамических режимов движения технических объектов, которые подвержены колебательным воздействиям. Используются методы математического моделирования, свойственные линейной теории колебаний и теории автоматического управления, для оценки, контроля и формирования состояний механических колебательных систем. Такие системы отражают существенные признаки машин и технологического оборудования в условиях вибрационного нагружения.

Для составления математических моделей, представляющих собой совокупность системы дифференциальных уравнений, алгебраическую систему и структурную схему, используется формализм Лагранжа, интегральные преобразования Лапласа и методы преобразования структурных схем.

Разработан метод и аналитический инструментарий построения математических моделей с учетом реализации режимов динамического гашения колебаний в механических системах с двумя степенями свободы при одновременном совместном действии двух внешних связанных нагружений.

Получены оригинальные результаты, отражающие особенности возникновения режимов динамического гашения; изучена их зависимость от условий связности внешних гармонических возмущений; приведены результаты численного моделирования.

Ключевые слова: механическая колебательная система, динамическое гашение колебаний, связность внешних сил, передаточные функции, устройства для преобразования движения
\end{abstract}

\section{Введение}

В последние годы вопросам безопасности эксплуатации и повышения надежности работы машин и оборудования, функционирующих в условиях высоких динамических нагрузок, уделяется большое внимание $[4,10,13]$. К числу таких технических средств относятся подвижной состав железнодорожного и автомобильного транспорта, технологические вибрационные машины, применяемые в различных отраслях промышленности $[7,11,13]$. Методы

() Елисеев А.В., Кузнецов Н.К., Елисеев С.В., 2021

Статья: поступила: 29.06.2021; рецензия: 05.07.2021; фринансирование: Иркутский государственный университет путей сообщения. 
и аналитический инструментарий определения особенностей режимов движения объектов транспортного и технологического назначения предопределяют внимание к стадиям «жизненного цикла» разработки современных машин и оборудования $[2,8]$.

Обеспечение соответствующих параметров динамического качества машин инициирует развитие научно-методологического базиса современной динамики машин, что связано с использованием методов системного анализа и аналитического аппарата теории систем. Развитие таких методологических позиций особенно характерно для технических средств, оснащенных системами автоматического управления, работа которых построена на использовании информации о динамическом состоянии объектов $[3,5,6]$.

Особое значение в оценке динамических состояний и возможностей их удержания в определённых границах имеет построение соответствующих принципиальных и расчетных схем, отображающих характерные особенности взаимодействия элементов систем.

Во многих случаях механические колебательные системы с поступательными и вращательными формами движения отображают существенные особенности динамики транспортных и технологических вибрационных машин, и поэтому используются в качестве расчетных схем соответствующих технических объектов. На начальном этапе рассматриваются системы с сосредоточенными параметрами, с небольшим количеством степеней свободы и малыми амплитудами колебаний относительно положения статического равновесия или установившегося состояния $[1,12,14]$.

Упрощение расчетных схем на первоначальных этапах исследования и оценок позволяет уточнить специфику динамических взаимодействий основных элементов и особенностей возникающих связей. Многие вопросы такого рода подходов нашли отражение в $[4,13]$.

Развитие методов математического моделирования позволяет решать многие практические задачи защиты машин, оборудования и приборов от вибрационных воздействий; вместе с тем расширяется спектр возможностей контроля, коррекции и формирования динамических состояний объектов при вибрационных возмущениях.

Можно полагать, что такое развитие происходит в результате расширения представлений о понятии дополнительных связей, реализуемых в механических колебательных системах не только посредством упругих и диссипативных элементов, но и с помощью более сложных образований на уровне использования простейших механизмов. Некоторые вопросы структурного анализа механических колебательных систем с учетом развития понятия дополнительной связи представлены в работах [12-14].

Дополнительные связи, в рамках таких подходов, могут быть реализованы на основе использования винтовых несамотормозящихся механизмов, а также зубчатых рычажных механизмов и др. [4, 13].

Внимание к дополнительным связям предопределяется новыми эффектами, специфическими особенностями движения и формами взаимодействия элементов механических колебательных систем.

В предлагаемой статье в рамках методов структурного математического моделирования развивается системный подход к анализу механических колебательных систем с учетом устройства для преобразования движения в форме винтового рычажного несамотормозящегося механизма.

\section{Методы}

Основные положения. Постановка задачи исследования. Рассматривается упругодиссипативная механическая колебательная система (рис. 1) с двумя степенями свободы с встроенными устройствами преобразования движения $L_{1}, L_{0}, L_{2}$.

Особенностью рассматриваемой системы является использование структурных элементов, обозначаемых как устройства для преобразования движения (УПД) с параметрами $L_{0}$, $L_{1}, L_{2}$, характеризующими значения приведенных массо-инерционных свойств вводимых нетрадиционных типовых элементов. Введение таких элементов формирует в колебательных 
структурах дополнительные связи, которые могут существенно влиять на динамическое состояние систем при возбуждении как свободных, так и вынужденных колебаний элементов механических колебательных систем.

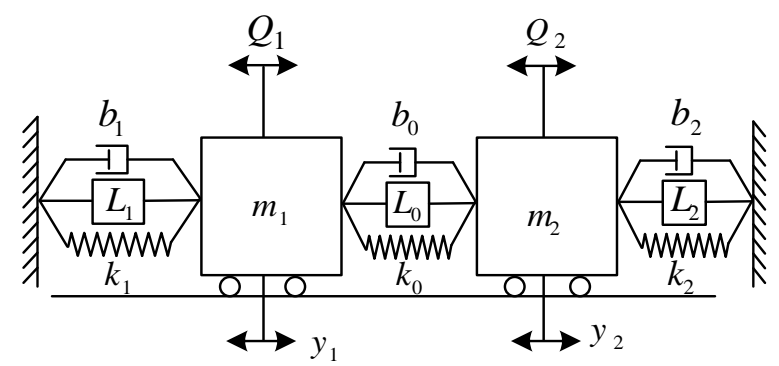

Рис. 1. Механическая колебательная система с учетом вязкого трения

Такие дополнительные связи могут быть реализованы, например, на основе использования рычажных или винтовых несамотормозящихся и механизмов (рис. $2, a, \sigma)$.
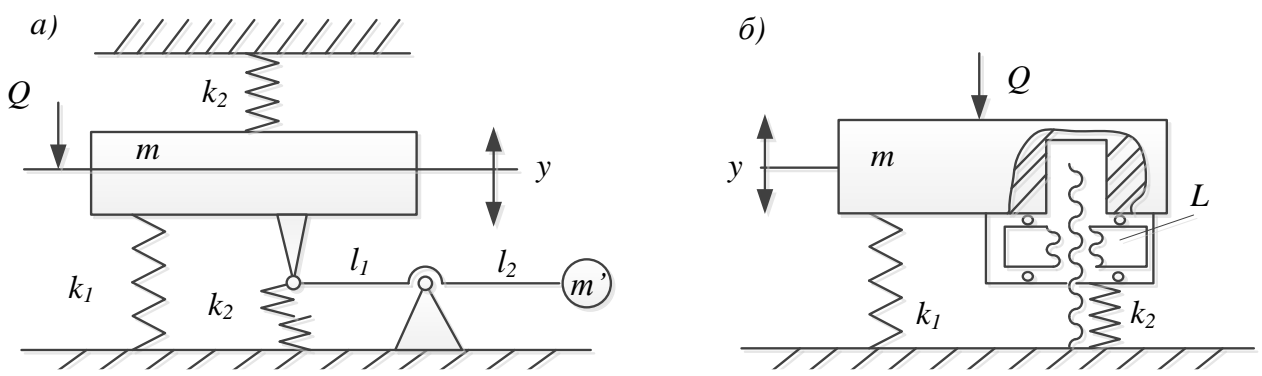

Рис. 2. Принципиальные схемы механических колебательных структур с дополнительными связями на основе:

а - рычажного механизма, б - винтового несамотормозящегося механизма

Массо-инерционные свойства связи, привносимые рычажным механизмом, определяются приведенной массой:

$$
L=m^{\prime}\left(\frac{l_{2}}{l_{1}}\right)^{2} \text {. }
$$

В свою очередь, дополнительная связь на основе винтового несамотормозящегося механизма может быть определена, в плане оценки массо-инерционных свойств, выражением

$$
L=\frac{J}{r_{c p} t^{2} \alpha},
$$

где $J$ - момент инерции гайки-маховика,

$r_{\mathrm{cp}}-$ радиус винта, $\alpha$ - угол наклона винтовой линии.

Устройства для преобразования движения могут быть построены также на основе других механизмов, например зубчатых. Особенности динамики механических колебательных систем с учетом УПД частично отражены в $[4,13]$.

Система (рис. 1) совершает вынужденные колебания под действием внешних гармонических возмущений $Q_{1}$ (приложенного к элементус массой $m_{1}$ ) и $Q_{2}$ (приложенного к элементу с массой $\left.m_{2}\right)$. Обобщенные координаты $y_{1}, y_{2}$ обозначают смещение исходной системы относительно положения статического равновесия. Кинетическая, потенциальная энергии и функция диссипации энергии определяются следующим образом:

$$
\begin{aligned}
& T=\frac{1}{2} m_{1} \dot{y}_{1}^{2}+\frac{1}{2} m_{2} \dot{y}_{2}^{2}+\frac{1}{2} L_{1} \dot{y}_{1}^{2}+\frac{1}{2} L_{0}\left(\dot{y}_{2}-\dot{y}_{1}\right)^{2}+\frac{1}{2} L_{2} \dot{y}_{2}^{2}, \\
& \Pi=\frac{1}{2} k_{1} y_{1}^{2}+\frac{1}{2} k_{2} y_{2}^{2}+\frac{1}{2} k_{0}\left(y_{2}-y_{1}\right)^{2},
\end{aligned}
$$




$$
F=\frac{1}{2} b_{1} \dot{y}_{1}^{2}+\frac{1}{2} b_{0}\left(\dot{y}_{2}-\dot{y}_{1}\right)^{2}+\frac{1}{2} b_{2} \dot{y}_{2}^{2} .
$$

Система уравнений Лагранжа второго рода после преобразования Лапласа может быть приведена к алгебраической форме:

$$
\left[\begin{array}{ll}
A_{11}(p) & A_{12}(p) \\
A_{21}(p) & A_{22}(p)
\end{array}\right]\left[\begin{array}{l}
\bar{y}_{1} \\
\bar{y}_{2}
\end{array}\right]=\left[\begin{array}{l}
\bar{Q}_{1} \\
\bar{Q}_{2}
\end{array}\right]
$$

где

$$
\begin{aligned}
& A_{11}(p)=\left(m_{1}+L_{1}+L_{0}\right) p^{2}+\left(b_{0}+b_{1}\right) p+k_{0}+k_{1} \\
& A_{21}(p)=-L_{0} p^{2}-b_{0} p-k_{0} \\
& A_{12}(p)=-L_{0} p^{2}-b_{0} p-k_{0} \\
& A_{22}(p)=\left(m_{2}+L_{2}+L_{0}\right) p^{2}+\left(b_{0}+b_{2}\right) p+k_{0}+k_{2} .
\end{aligned}
$$

В конечном итоге уравнения (6) принимают вид

$$
\left[(M+L) p^{2}+B p+C\right] \vec{Y}=\vec{Q},
$$

где $p=\sigma+j \omega-$ комплексная переменная Лапласа, $j=\sqrt{ }-1, M-$ матрица массо-инерционных коэффициентов, $L$ - матрица коэффициентов устройств преобразования движений, $B$ - матрица коэффициентов вязкого трения, $C$ - матрица коэффициентов жесткости:

$$
M=\left[\begin{array}{cc}
m_{1} & 0 \\
0 & m_{2}
\end{array}\right], L=\left[\begin{array}{cc}
L_{1}+L_{0} & -L_{0} \\
-L_{0} & L_{2}+L_{0}
\end{array}\right] B=\left[\begin{array}{cc}
b_{0}+b_{1} & -b_{0} \\
-b_{0} & b_{0}+b_{2}
\end{array}\right], C=\left[\begin{array}{cc}
k_{0}+k_{1} & -k_{0} \\
-k_{0} & k_{0}+k_{2}
\end{array}\right] .
$$

Задача заключается в разработке в рамках системного подхода методов структурного математического моделирования, позволяющих формировать, оценивать и контролировать специфические режимы механических колебательных систем, содержащих в своей структуре устройства для преобразований движений.

\section{Моделирование}

Особенности режимов динамического гашения с учетом связности внешнего воздействия. Полагается, что для внешних возмущений $Q_{1}$ и $Q_{2}$ выполняется соотношение

$$
Q_{2}=\alpha Q_{1},
$$

где $\alpha$ - коэффициент связности внешних возмущений. Физический смысл условия (10) может, к примеру, отражать связь амплитуд колебаний силовых возмущений со стороны двух синхронно работающих вибраторов, установленных в различных точках рабочего органа вибрационной технологической машины.

Система линейных алгебраических уравнений (8) позволяет механической колебательной системе (рис. 1) сопоставить структурную схему эквивалентной в динамическом отношении системы автоматического управления [13]. Структурная математическая модель (рис. 3 ) состоит из двух парциальных подсистем и имеет два внешних воздействия $Q_{1}$ и $Q_{2}$, связанных соотношением (10).

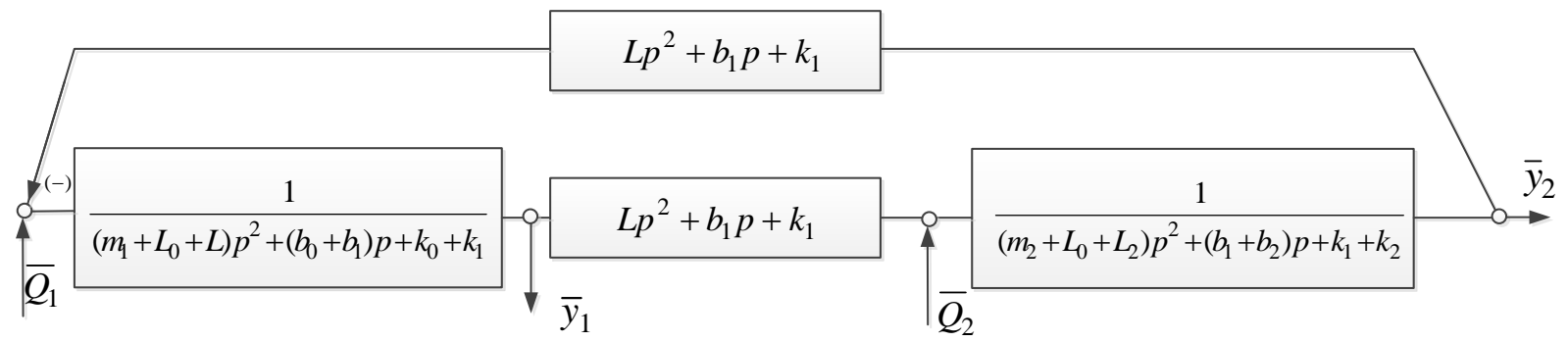

Рис. 3. Структурная математическая модель (структурная модель), соответствующая расчетной схеме на рис. 1. 
На структурной схеме (рис. 3) приняты обозначения: $p=\sigma+j \omega-$ комплексная переменная Лапласа, значок $<->$ на переменной означает её изображение по Лапласу. Использование структурной схемы даёт возможность в подходах системного анализа использовать аналитический аппарат теории автоматического управления $[6,9,10]$.

Особенности режима динамического гашения колебаний по координате $\overline{y_{1}}$. Режим динамического гашения по координате $\bar{y}_{1}$ может быть определен на основе передаточной функции системы

$$
\left.W_{11}(\alpha, p)\right|_{Q_{2}=\alpha Q_{1}}=\frac{\bar{y}_{1}(\alpha)}{\bar{Q}_{1}},
$$

где приложенная сила $Q_{1}$ рассматривается как входной сигнал, а движение системы по координате $\overline{y_{1}}-$ как выходной сигнал.

Движение системы по координате $\overline{y_{1}}$ в преобразованиях Лапласа, если полагать возникающие связи переменными, отображаются формулами Крамера:

$$
\bar{y}_{1}=\frac{\left|\begin{array}{ll}
\overline{Q_{1}} & A_{12}(p) \\
\alpha \overline{Q_{1}} & A_{22}(p)
\end{array}\right|}{A(p)},
$$

где $A(p)=A_{11}(p) A_{22}(p)-A_{21}(p) A_{12}(p)-$ характеристический многочлен системы (частотное характеристическое уравнение). Необходимо отметить, что в общем случае соответствующее характеристическое уравнение $A(p)=0$ имеет корни $p_{1}, p_{2}, p_{3}, p_{4}$. В случае пренебрежимо малых сил трения $(B=0)$ характеристическое уравнение вырождается в биквадратное уравнение $A_{l}\left(p^{2}\right)=0$, разрешение которого после использования $p=j \omega$ позволяет определить собственные частоты системы $\omega_{01}, \omega_{02}$.

Аналитическая форма выражения для передаточной функции в зависимости от комплексного параметра $p$ и параметра связности внешнего воздействия $\alpha$ имеет вид

$$
\left.W_{11}(\alpha, p)\right|_{Q_{2}=\alpha Q_{1}}=\frac{A_{22}(p)-\alpha A_{12}(p)}{|A(p)|} .
$$

Определение частоты динамического гашения колебаний по координате $\bar{y}_{1}$ при условии несовпадения частоты динамического гашения с собственными частотами возможно из равенства нулю числителя (13):

$$
\left(m_{2}+L_{2}+L_{0}\right) p^{2}+\left(b_{0}+b_{2}\right) p+k_{0}+k_{2}+\alpha\left(L_{0} p^{2}+b_{0} p+k_{0}\right)=0 .
$$

Подобного рода задачи могут возникать при оценке устойчивости динамических состояний при сближении частоты динамического гашения колебаний с собственной частотой.

Обобщенный подход с учетом сил трения предполагает определение комплексного параметра в форме $p=\sigma+j \omega$ и $p^{2}=\sigma^{2}-\omega^{2}+j 2 \sigma \omega$, удовлетворяющего условиям $A_{22}(p)-\alpha A_{12}(p)=0$ в виде системы уравнений:

$$
\begin{aligned}
& \left(m_{2}+L_{2}+L_{0}+\alpha L_{0}\right)\left(\sigma^{2}-\omega^{2}\right)+\left(b_{0}+b_{2}+\alpha b_{0}\right) \sigma+k_{0}+k_{2}+\alpha k_{0}=0 \\
& \left(m_{2}+L_{2}+L_{0}+\alpha L_{0}\right) 2 \sigma \omega+\left(b_{0}+b_{2}+\alpha b_{0}\right) \omega=0 .
\end{aligned}
$$

При пренебрежимо малых силах трения $\left(b_{0}+b_{2}=0\right)$ условия $(15),(16)$ принимают вид

$-\omega^{2}\left(m_{2}+L_{2}+L_{0}(1+\alpha)\right)+k_{0}(1+\alpha)+k_{2}=0$.

Следовательно, в предположении $b_{0}+b_{2}=0$, частота динамического гашения колебаний $\omega_{11, \text { dyn }}$ по координате $\overline{y_{1}}$ определяется формулой

$$
\omega_{11, d y n}^{2}(\alpha)=\frac{k_{0}(1+\alpha)+k_{2}}{m_{2}+L_{2}+L_{0}(1+\alpha)} .
$$

Таким образом, частота динамического гашения колебаний $\omega_{11}$ зависит от параметров $m_{2}, L_{2}, L_{0}, k_{0}, k_{2}$ и параметра связности $\alpha$. При условии несовпадения $\omega_{11}$ с собственными частотами $\omega_{01}, \omega_{02}$ системы обеспечивается динамическое гашение колебаний по координате $\bar{y}_{1}$. 
Особенности режима динамического гашения по координате $\overline{y_{2}}$. Для определения режима динамического гашения колебаний по координате $\overline{y_{2}}$ можно воспользоваться передаточной функцией системы $W_{12}$, которая определяется выражением

$$
\left.W_{12}(p, \alpha)\right|_{Q_{2}=0}=\frac{\bar{y}_{2}(\alpha)}{\bar{Q}_{1}},
$$

где приложенная сила $\overline{Q_{1}}$ рассматривается как входной сигнал, а движение системы по координате $\overline{y_{2}}-$ как выходной сигнал.

Особенности движения системы по координате $\overline{y_{2}}$ в преобразованиях Лапласа могут быть учтены применением формул Крамера:

$$
\bar{y}_{2}=\frac{\left|\begin{array}{ll}
A_{11}(p) & \bar{Q}_{1} \\
A_{21}(p) & \alpha \bar{Q}_{1}
\end{array}\right|}{A(p)} .
$$

В свою очередь соответствующая передаточная функция (19) в зависимости от комплексного параметра $p$ принимает вид

$$
\left.W_{12}(p, \alpha)\right|_{Q_{2}=\alpha Q_{1}}=\frac{\alpha A_{11}(p)-A_{21}(p)}{|A(p)|} .
$$

Определение частоты динамического гашения колебаний $\omega_{12}$ по координате $\overline{y_{2}}$ в предположении, что эта частота не совпадает с собственной частотой системы, возможно из условия $\alpha A_{11}(p)-A_{21}(p)=0$ :

$$
\alpha\left(\left(m_{1}+L_{1}+L_{0}\right) p^{2}+\left(b_{0}+b_{1}\right) p+k_{0}+k_{1}\right)+\left(L_{0} p^{2}+b_{0} p+k_{0}\right)=0 .
$$

Обобщенный подход с учетом сил трения предполагает определение комплексного параметра в форме $p=\sigma+j \omega$ и $p^{2}=\sigma^{2}-\omega^{2}+j 2 \sigma \omega$, удовлетворяющего условиям $\alpha A_{11}-A_{2 l}=0$ в виде системы уравнений:

$$
\begin{aligned}
& \left(\alpha\left(m_{1}+L_{l}+L_{0}\right)+L_{0}\right)\left(\sigma^{2}-\omega^{2}\right)+\left(\alpha\left(b_{0}+b_{1}\right)+b_{0}\right) \sigma+\alpha\left(k_{0}+k_{l}\right)+k_{0}=0, \\
& \left(\alpha\left(m_{1}+L_{1}+L_{0}\right)+L_{0}\right) 2 \sigma \omega+\left(\alpha\left(b_{0}+b_{1}\right)+b_{0}\right) \omega=0 .
\end{aligned}
$$

При пренебрежимо малых силах трения $(B=0, \sigma=0)$ условия $(23),(24)$ принимают вид

$$
-\omega^{2}\left(\alpha\left(m_{1}+L_{l}+L_{0}\right)+L_{0}\right)+\alpha\left(k_{0}+k_{l}\right)+k_{0}=0 .
$$

Следовательно, в предположении $(B=0, \sigma=0)$ частота динамического гашения колебаний по координате $\overline{y_{2}}$ может быть определена выражением

$$
\omega_{12, d y n}^{2}=\frac{\alpha\left(k_{0}+k_{1}\right)+k_{0}}{\alpha\left(m_{1}+L_{1}+L_{0}\right)+L_{0}} .
$$

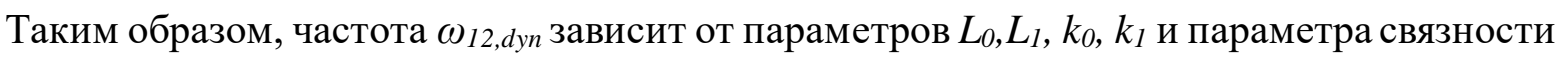
внешних сил $\alpha$. При условии несовпадения $\omega_{12, d y n}$ с собственными частотами $\omega_{01}, \omega_{02}$ системы обеспечивается динамическое гашение колебаний по координате $\overline{y_{2}}$.

Таким образом, при учете действия одиночной силы режимы динамического гашения колебаний могут ожидаться на тех массо-инерционных элементах, к которым приложена внешняя сила (внешняя сила может быть приложена либо к элементу $m_{1}$, либо к элементу $m_{2}$ ). Наличие в системе дополнительных связей (устройство для преобразования движения) вносит особенности в режим динамического гашения колебаний по каждому из элементов, но не приводит к совместному гашению колебаний по двум координатам (для системы, в которой обобщенные силы не равны нулю).

\section{Сближение частот динамического гашения колебаний}

Рассматривается возможность совпадения частот динамического гашения по координатам $\overline{y_{1}}, \overline{y_{2}}$ в результате выбора специальных значений параметров связности внешних сил. Пусть частоты динамического гашения совпадают: 
$\omega_{12, d y n}=\omega_{11, d y n}$.

Это означает, что для параметров системы выполняется соотношение

$$
\frac{k_{0}+k_{2}+\alpha k_{0}}{m_{2}+L_{2}+L_{0}+\alpha L_{0}}=\frac{\alpha\left(k_{0}+k_{1}\right)+k_{0}}{\alpha\left(m_{1}+L_{1}+L_{0}\right)+L_{0}} .
$$

Уравнение (27) имеет два корня - $\alpha_{1}$ и $\alpha_{1}$, для которых выполнено совпадение частот динамического гашения колебаний $\omega_{12, d y n}=\omega_{11, d y n}$ :

$$
\begin{aligned}
& \frac{k_{0}+k_{2}+\alpha_{1} k_{0}}{m_{2}+L_{2}+L_{0}+\alpha_{1} L_{0}}=\omega_{1}{ }^{2}, \frac{\alpha_{1}\left(k_{0}+k_{1}\right)+k_{0}}{\alpha_{1}\left(m_{1}+L_{1}+L_{0}\right)+L_{0}}=\omega_{1}^{2}, \\
& \frac{k_{0}+k_{2}+\alpha_{2} k_{0}}{m_{2}+L_{2}+L_{0}+\alpha_{2} L_{0}}=\omega_{2}{ }^{2}, \frac{\alpha_{2}\left(k_{0}+k_{1}\right)+k_{0}}{\alpha_{2}\left(m_{1}+L_{1}+L_{0}\right)+L_{0}}=\omega_{2}{ }^{2} .
\end{aligned}
$$

Вместе с тем одновременное совпадение частот динамического гашения $\omega_{12, d y n}=\omega_{11, d y n}$ означает одновременное выполнение условий $A_{22}(p)-\alpha A_{12}(p)=0$ и $\alpha A_{11}(p)-A_{21}(p)=0$ для значений соответствующих величин $\alpha_{1}, \alpha_{1}, \omega_{1}, \omega_{1}$ :

$A_{22}\left(j \omega_{1}\right)-\alpha_{1} A_{12}\left(j \omega_{1}\right)=0, \alpha_{1} A_{11}\left(j \omega_{1}\right)-A_{21}\left(j \omega_{1}\right)=0$,

$A_{22}\left(j \omega_{2}\right)-\alpha_{2} A_{12}\left(j \omega_{2}\right)=0, \alpha_{2} A_{11}\left(j \omega_{2}\right)-A_{21}\left(j \omega_{2}\right)=0$.

Выражения (31) и (32) могут быть представлены в виде

$$
\begin{aligned}
& \frac{A_{22}\left(j \omega_{1}\right)}{A_{12}\left(j \omega_{1}\right)}=\frac{A_{21}\left(j \omega_{1}\right)}{A_{11}\left(j \omega_{1}\right)}, \\
& \frac{A_{22}\left(j \omega_{2}\right)}{A_{12}\left(j \omega_{2}\right)}=\frac{A_{21}\left(j \omega_{2}\right)}{A_{11}\left(j \omega_{2}\right)} .
\end{aligned}
$$

Выполнение равенств (33), (34) означает, что характеристический многочлен $A(j \omega)$ системы равен нулю на частотах $\omega_{1} u \omega_{2}$. Таким образом, если частоты динамического гашения колебаний $\omega_{12, d y n}=\omega_{11, d y n}$ совпадают между собой, то они совпадают и с частотой собственных колебаний.

\section{Особенности режимов сближения частот динамического гашения}

Рассматривается вариант предельного сближения частот динамического гашения путем вариации коэффициента связности внешних возмущений.

Приведенные выкладки позволяют заключить, что наличие двух внешних сил, связанных между собой коэффициентом $\alpha$, не означает того, что в системе может быть реализован режим одновременного динамического гашения колебаний на одной частоте.

Если коэффициент связности внешних сил равен $\alpha_{1}$, то возможно выполнение следующих соотношений:

$A_{22}\left(j \omega_{1}\right)-\alpha_{1} A_{12}\left(j \omega_{1}\right)=0, \quad \alpha_{1} A_{11}\left(j \omega_{1}\right)-A_{21}\left(j \omega_{1}\right)=0$,

$A_{22}\left(j \omega_{2}\right)-\alpha_{1} A_{12}\left(j \omega_{2}\right) \neq 0, \alpha_{1} A_{11}\left(j \omega_{2}\right)-A_{21}\left(j \omega_{2}\right) \neq 0$.

Передаточная функция $W_{11}\left(p, \alpha_{1}\right)$ в данном случае может быть представлена в виде

$$
\left.W_{11}\left(\alpha_{1}, j \omega\right)\right|_{Q_{2}=\alpha_{1} Q_{1}}=\frac{\left|\begin{array}{cc}
1 & A_{12}(j \omega) \\
\alpha_{1} & A_{22}(j \omega)
\end{array}\right|}{\left|\begin{array}{ll}
A_{11}(j \omega) & A_{12}(j \omega) \\
A_{21}(j \omega) & A_{22}(j \omega)
\end{array}\right|} .
$$

После подстановки

$$
\alpha_{1}=\frac{A_{21}\left(j \omega_{1}\right)}{A_{11}\left(j \omega_{1}\right)}
$$

выражение (37) приводится к форме 


$$
\left.W_{11}\left(\alpha_{1}, j \omega\right)\right|_{Q_{2}=\alpha_{1} Q_{1}}=\frac{1}{A_{11}\left(j \omega_{1}\right)} \frac{\left|\begin{array}{ll}
A_{11}\left(j \omega_{1}\right) & A_{12}(j \omega) \\
A_{21}\left(j \omega_{1}\right) & A_{22}(j \omega)
\end{array}\right|}{\left|\begin{array}{ll}
A_{11}(j \omega) & A_{12}(j \omega) \\
A_{21}(j \omega) & A_{22}(j \omega)
\end{array}\right|} .
$$

Аналогично передаточная функция $W_{12}\left(p, \alpha_{1}\right)$ может быть представлена выражением

$$
\left.W_{12}\left(\alpha_{1}, j \omega\right)\right|_{Q_{2}=\alpha_{1} Q_{1}}=\frac{\left|\begin{array}{ll}
A_{11}(j \omega) & 1 \\
A_{21}(j \omega) & \alpha_{1}
\end{array}\right|}{\left|\begin{array}{ll}
A_{11}(j \omega) & A_{12}(j \omega) \\
A_{21}(j \omega) & A_{22}(j \omega)
\end{array}\right|} .
$$

После подстановки

$$
\alpha_{1}=\frac{A_{22}\left(j \omega_{1}\right)}{A_{12}\left(j \omega_{1}\right)}
$$

в выражение (40) получим, что

$$
\left.W_{12}\left(\alpha_{1}, j \omega\right)\right|_{Q_{2}=\alpha_{1} Q_{1}}=\frac{1}{A_{12}\left(j \omega_{1}\right)} \frac{\left|\begin{array}{ll}
A_{11}(j \omega) & A_{12}\left(j \omega_{1}\right) \\
A_{21}(j \omega) & A_{22}\left(j \omega_{1}\right)
\end{array}\right|}{\left|\begin{array}{ll}
A_{11}(j \omega) & A_{12}(j \omega) \\
A_{21}(j \omega) & A_{22}(j \omega)
\end{array}\right|} .
$$

Если коэффициент связности внешних сил равен $\alpha_{2}$, то это предопределяет выполнение следующих соотношений:

$A_{22}\left(j \omega_{2}\right)-\alpha_{2} A_{12}\left(j \omega_{2}\right)=0, \quad \alpha_{2} A_{11}\left(j \omega_{2}\right)-A_{21}\left(j \omega_{2}\right)=0$,

$A_{22}\left(j \omega_{1}\right)-\alpha_{2} A_{12}\left(j \omega_{2}\right) \neq 0, \alpha_{2} A_{11}\left(j \omega_{1}\right)-A_{21}\left(j \omega_{1}\right) \neq 0$.

Передаточная функция $W_{11}\left(p, \alpha_{2}\right)$ может быть также представлена в виде

$$
\left.W_{11}\left(\alpha_{2}, j \omega\right)\right|_{Q_{2}=\alpha_{2} Q_{1}}=\frac{\left|\begin{array}{cc}
1 & A_{12}(j \omega) \\
\alpha_{2} & A_{22}(j \omega)
\end{array}\right|}{\left|\begin{array}{ll}
A_{11}(j \omega) & A_{12}(j \omega) \\
A_{21}(j \omega) & A_{22}(j \omega)
\end{array}\right|},
$$

что после подстановки

$$
\alpha_{2}=\frac{A_{21}\left(j \omega_{2}\right)}{A_{11}\left(j \omega_{2}\right)}
$$

в выражение (45) определяется выражением

$$
\left.W_{11}\left(\alpha_{2}, j \omega\right)\right|_{Q_{2}=\alpha_{2} Q_{1}}=\frac{1}{A_{11}\left(j \omega_{2}\right)} \frac{\left|\begin{array}{ll}
A_{11}\left(j \omega_{2}\right) & A_{12}(j \omega) \\
A_{21}\left(j \omega_{2}\right) & A_{22}(j \omega)
\end{array}\right|}{\left|\begin{array}{ll}
A_{11}(j \omega) & A_{12}(j \omega) \\
A_{21}(j \omega) & A_{22}(j \omega)
\end{array}\right|} .
$$

Передаточная функция $W_{12}\left(\alpha_{2}, p\right)$ может быть представлена также в виде

$$
\left.W_{12}\left(\alpha_{2}, j \omega\right)\right|_{Q_{2}=\alpha_{2} Q_{1}}=\frac{\left|\begin{array}{ll}
A_{11}(j \omega) & 1 \\
A_{21}(j \omega) & \alpha_{2}
\end{array}\right|}{\left|\begin{array}{ll}
A_{11}(j \omega) & A_{12}(j \omega) \\
A_{21}(j \omega) & A_{22}(j \omega)
\end{array}\right|} .
$$

После соответствующей подстановки

$$
\alpha_{2}=\frac{A_{22}\left(j \omega_{2}\right)}{A_{12}\left(j \omega_{2}\right)}
$$

выражение (48) преобразуется к виду 


$$
\left.W_{12}\left(\alpha_{2}, j \omega\right)\right|_{Q_{2}=\alpha_{2} Q_{1}}=\frac{1}{A_{12}\left(j \omega_{2}\right)} \frac{\left|\begin{array}{ll}
A_{11}(j \omega) & A_{12}\left(j \omega_{2}\right) \\
A_{21}(j \omega) & A_{22}\left(j \omega_{2}\right)
\end{array}\right|}{\left|\begin{array}{ll}
A_{11}(j \omega) & A_{12}(j \omega) \\
A_{21}(j \omega) & A_{22}(j \omega)
\end{array}\right|} .
$$

Выражения (42) и (50) представляют собой амплитудно-частотные характеристики, являющиеся результатом разрешения неопределенности при сближении амплитуд динамического гашения колебаний с одной из собственных частот, которые в соответствующих точках непрерывны, что позволяет рассматривать данное сочетание параметров как специфический режим преобразования системы с двумя степенями свободы в систему с одной степенью свободы.

Таким образом, при изучении возможности достижения режима одновременного совместного динамического гашения колебаний изменения коэффициента связности внешних сил оказывает существенное влияние на формирование динамических состояний системы.

\section{Результаты, их обсуждение}

Некоторые результаты вычислительного моделирования. Рассматривается механическая колебательная система, представленная на расчетной схеме (рис. 1) с параметрами $m_{1}=2$ кг, $m_{2}=1$ кг, $k_{1}=1 \mathrm{H} / \mathrm{M}, k_{0}=4 \mathrm{H} / \mathrm{M}, k_{2}=3 \mathrm{H} / \mathrm{M}, L_{1}=1$ кг, $L_{2}=2$ кг, $L_{0}=3$ кг. Расчетные частоты собственных колебаний составляют соответственно $\omega_{1}=0.7713$ рад/c, $\omega_{2}=1.0875$ рад/с. Коэффициенты связности внешних силовых возмущений определяются значениями $\alpha_{1}=-1.5485$, $\alpha_{2}=0.2152$. Представлены (рис. 4) амплитудно-частотные характеристики (АЧХ) для передаточных функций $W_{11}(p), W_{12}(p)$ для коэффициента связности $\alpha_{1}=-1.5485$. Учитывая формы графиков, отметим, что на частоте собственных колебаний $\omega_{1}$ функция АЧХ непрерывна и принимает положительные значения. Вместе с тем на собственной частоте $\omega_{2}$ функции АЧХ имеют разрывы второго рода, отображающие особенности реализации резонанса. Данные графики показывают отсутствие резонанса по двум координатам на частоте собственных колебаний $\omega_{1}$. Вместе с тем не наблюдается и гашение колебаний, так как функции принимают конечные ненулевые значения.

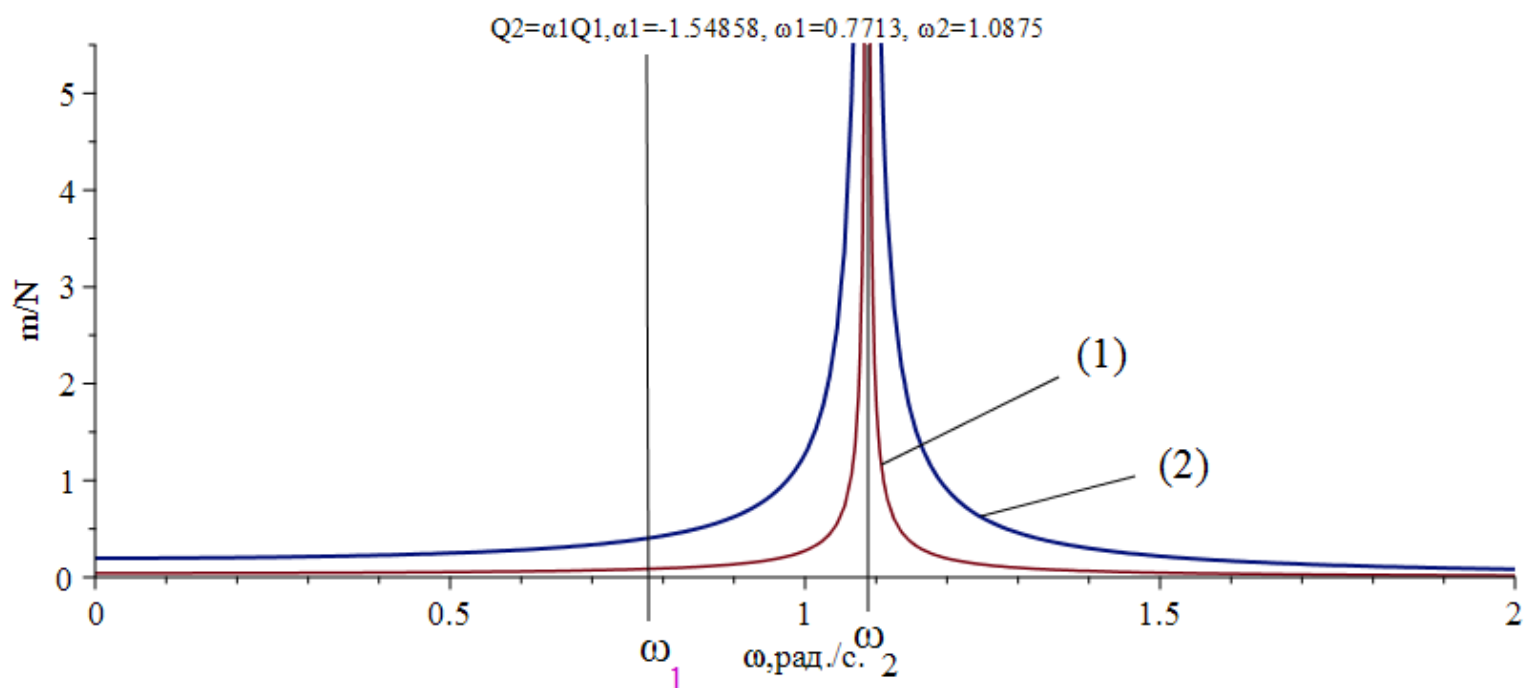

Рис 4. Амплитудно-частотные характеристики системы для коэффиициента связности $\alpha_{1}=-1.5485$ :

1 - $y_{1}$ - выходной сигнал, 2 - $y_{2}-$ выходной сигнал

Таким образом, при выборе параметра (в данном случае $\alpha$ ), обеспечивающего совпадения режимов динамического гашения колебаний по двум частотам одновременно (на одной частоте), в системе происходит перестройка, и она начинает работать как система с одной 
степенью свободы. Нетрадиционный подход в достижении специфического режима динамического гашения колебаний в системе с двумя степенями свободы при двух взаимосвязанных возмущениях приводит к появлениям эффекта «упрощения» структуры механической колебательной системы, демонстрирующей возможность при совпадении частот динамического гашения преобразоваться в систему с одной степенью свободы.

В свою очередь, потеря системой одной степени свободы находит своё отражение в вырождении амплитудно-частотной характеристики межпарциальной передаточной функции в константу, принимающую значения $A_{11}\left(j \omega_{1}\right) / A_{12}\left(j \omega_{1}\right)$ и $A_{11}\left(j \omega_{2}\right) / A_{12}\left(j \omega_{2}\right)$ для коэффициентов связности внешних силовых возмущений $\alpha_{1}$ и $\alpha_{2}$ соответственно.

Аналогично представлены АЧХ (рис. 5), полученные из передаточных функций $W_{11}(p)$, $W_{12}(p)$ при коэффициентах связности внешних сил $\alpha_{2}=0.2142$. На частоте собственных колебаний $\omega_{2}$ амплитудно-частотные характеристики непрерывны и принимают положительные значения, а на собственной частоте $\omega_{1}$ амплитудно-частотные характеристики демонстрируют реализацию резонанса.

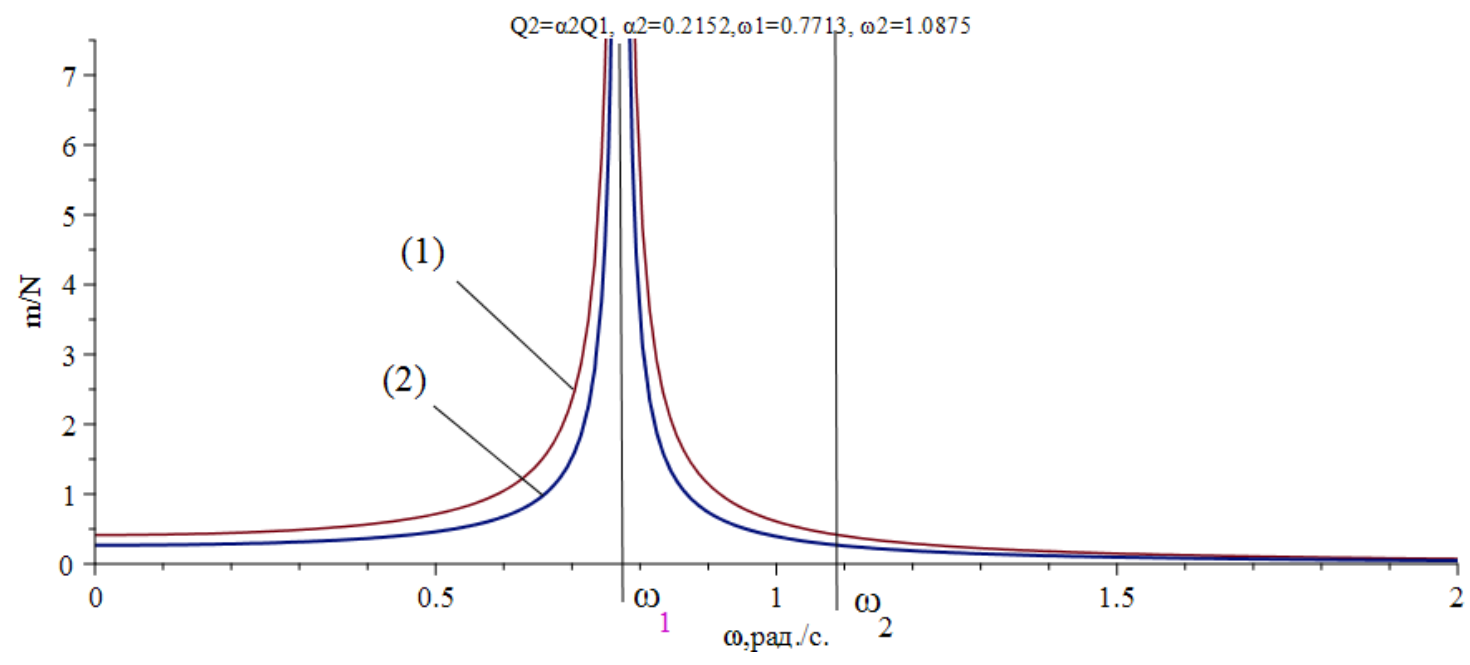

Рис. 5. Амплитудно-частотные характеристики системы для коэффрициента связности $\alpha_{2}=0.2152$ :

$1-\bar{y}_{1}-$ выходной сигнал, $2-\bar{y}_{2}-$ выходной сигнал

Рисунок 6 представляет амплитудно-частотные характеристики по двум координатам для параметра связности внешних воздействий $\alpha=-1.4$, условно близкого к экстремальному параметру связности $\alpha_{1}$. На этом рисунке видны особенности «уменьшения» амплитуд колебания и резонанса на частоте $\omega_{1}$.

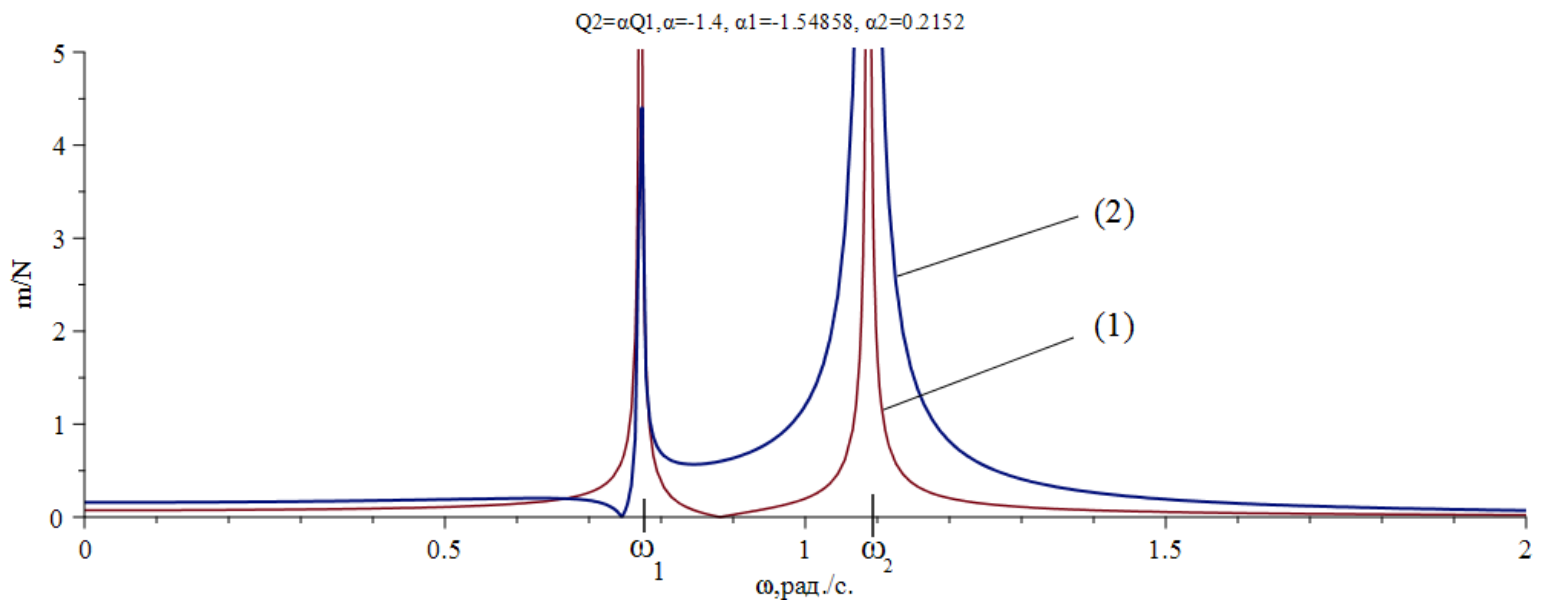

Рис. 6. Амплитудно-частотные характеристики системы для коэфффициента связности $\alpha=-1.4$ : $1-\bar{y}_{1}-$ входной сигнал, $2-\bar{y}_{2}-$ входной сигнал 
Аналогично представлены амплитудно-частотные характеристики (по двум координатам для параметра связности внешних воздействий $\alpha=0.4$, условно близкого к экстремальному параметру связности $\alpha_{2}$ ), отражающие особенности «уменьшения» резонанса на частоте $\omega_{2}$ (рис. 7).

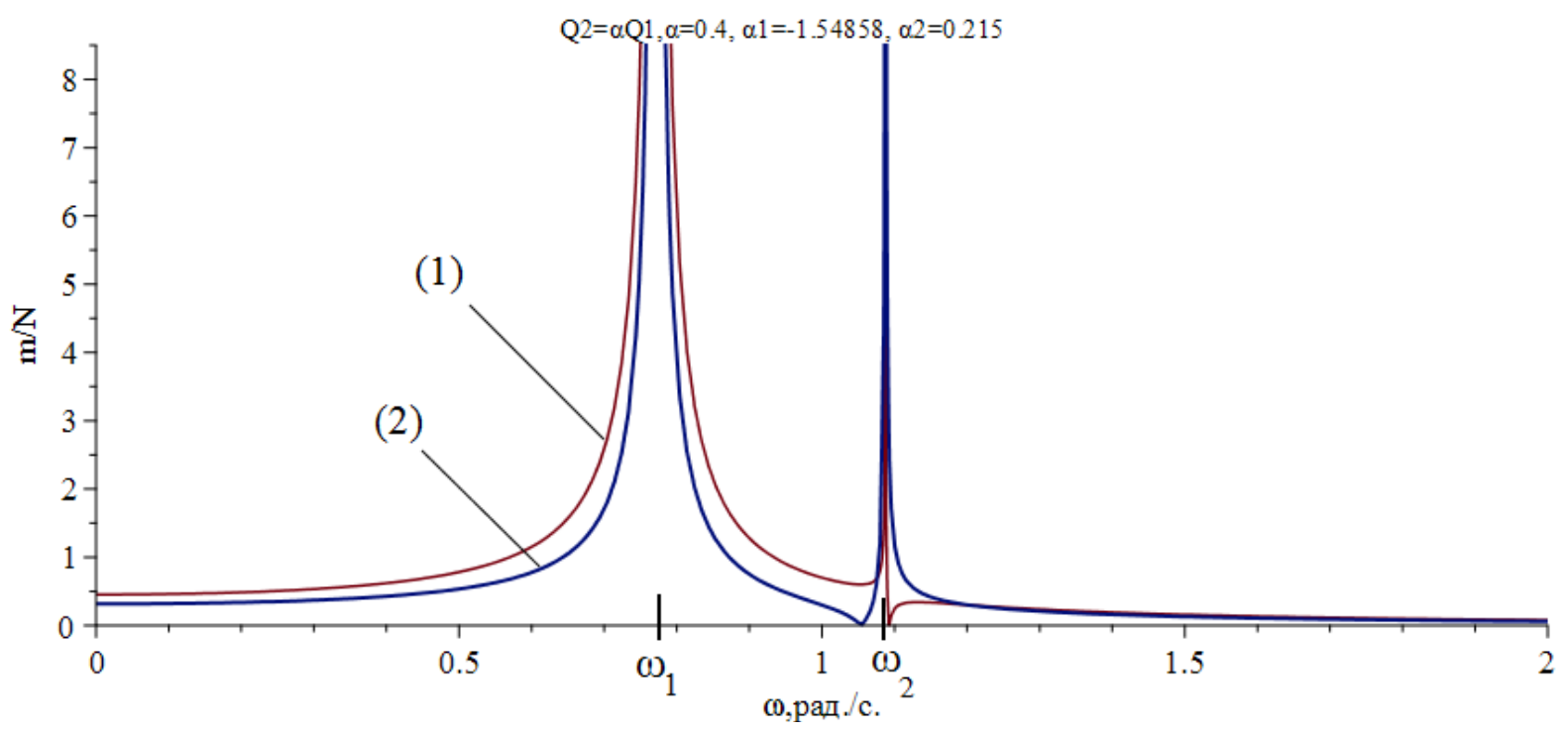

Рис. 7. Амплитудно-частотные характеристики системы для коэффрициента связности $\alpha=0.4$ : $1-\bar{y}_{1}-$ выходной сигнал, $2-\bar{y}_{2}-$ выходной сигнал

Вместе с тем представлены АЧХ по двум координатам для параметра связности внешних воздействий $\alpha=-0.4$ (рис. 8), условно удаленного от обоих экстремальных значений параметра связности $\alpha_{1}$ и $\alpha_{2}$. Видны (рис. 6) характерные особенности резонансных явлений.

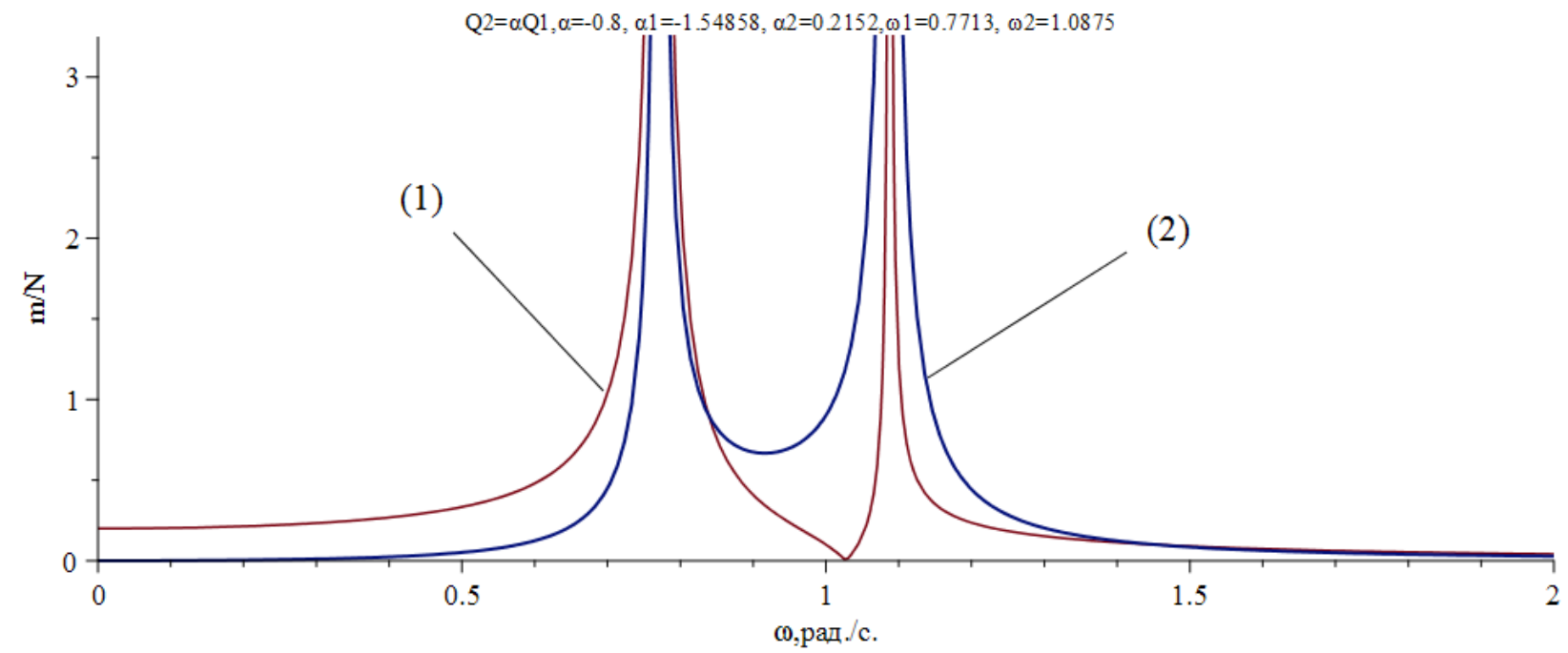

Рис. 8. Амплитудно-частотные характеристики системы для коэффрициента связности $\alpha=-0.8$ : $1-\bar{y}_{1}-$ входной сигнал, $2-\bar{y}_{2}-$ входной сигнал

\section{Выводы}

В работах, посвященных поиску частот совместного динамического гашения колебаний одновременно по двум координатам в механических колебательных системах, рассмотрен ряд подходов к определению особенностей специфических режимов. Детализированный анализ режимов «обнуления» обобщенных координат систем приводит к следующим выводам.

В цепной механической колебательной системе с двумя степенями свободы совместное действие гармонических внешних сил, приложенных к массо-инерционным элементам и находящихся в пропорциональной зависимости, выраженной с помощью параметра связности, не 
изменяет частоты собственных колебаний системы. Вместе с тем параметр связности внешних гармонических сил может рассматриваться как регулирующий фактор, однозначно определяющий две частоты динамического гашения, каждая из которых «обнуляет» одну из двух амплитуд колебаний массо-инерционных элементов в установившемся режиме движения.

Для определения режима совместного динамического гашения колебания двух массоинерционных элементов в режиме установившегося движения поставлена задача определения параметра связности внешних сил, для которого две частоты динамического гашения совпадают.

Показано, что варьирование коэффициента связности в направлении сближения между собой частот динамического гашения колебаний по разным координатам приводит к их приближению к собственным частотам.

Установлено, что для рассматриваемого класса механических колебательных систем существует два коэффициента связности внешних сил, таких, что при наличии двух собственных частот один из резонансов «гасится» в том смысле, что на одной из частот собственных колебаний амплитуды обеих координат принимают конечные, но ненулевые, значения. Таким образом, можно полагать, что для рассматриваемого класса систем режим «совместного» динамического гашения по двум координатам, в смысле обнуления их амплитуд, не реализуется.

Показано, что существует два коэффициента связности внешних сил, которые могут быть сопоставлены двум частотам собственных колебаний, таких, что при выборе внешнего силового воздействия с использованием коэффициента связности соответствующая амплитудно-частотная характеристика с двумя разрывами второго рода в точках резонанса вырождается в амплитудно-частотную характеристику с одной точкой разрыва второго рода. При детализированном рассмотрении особенностей динамических свойств выявлено, что при действии двух гармонических сил возможно динамическое гашение на частоте собственных колебаний, что ликвидирует процесс резонансных колебаний, но не исключает реализацию резонанса на второй резонансной частоте. Аналогичная ситуация реализуется для второй частоты резонанса.

Дальнейшие направления исследований представляют собой развитие понятий, связанных с динамическим гашением колебаний, передаточным отношением виртуального рычага, динамической приведенной жесткостью, связностью форм движений массо-инерционных элементов и внешних воздействий для систем с одной степенью свободы, имеющих поступательный, вращательный и винтовой характер колебаний.

Обобщение развиваемых понятий динамического гашения колебаний, передаточных отношений, динамической жесткости, связности форм движений элементов и внешних возмущений предполагает перенос разработанных представлений и методов структурного математического моделирования на механические колебательные системы с объектом в виде твердого тела.

Вклад авторов в статью: А.В. Елисеев - проведение аналитических расчетов и численных экспериментов, подготовка текста статьи, формирование выводов; Н.К. Кузнецов - анализ результатов исследований, консультации; С.В. Елисеев - формирование основной концепции, цели и задачи исследования. Авторы заявляют об отсутствии конфрликта интересов.

\section{СПИСОК ЛИТЕРАТУРЫ}

1. Ганиев Р.Ф., Кононенко В.О. Колебания твердых тел. М.: Наука, 1976. 432 с.

2. Доронин С.В., Шокин Ю.И., Лепихин А.М., Москвичев В.В. Моделирование прочности и разрушения несущих конструкций технических систем. Новосибирск: Наука, 2005. 249 с.

3. Елисеев А.В., Кузнецов Н.К., Московских А.О. Динамика машин. Системные представления, структурные схемы и связи элементов: монография. М.: Инновационное машиностроение, 2019. $381 \mathrm{c}$.

4. Елисеев С.В., Артюнин А.И. Прикладная теория колебаний в задачах динамики линейных механических систем: монография. Новосибирск: Наука, 2016. 459 с. 
5. Емельянов С.В., Коровин С.К. Новые типы обратной связи: управление при неопределенности. М.: Наука: Физматлит, 1997. 352 с.

6. Коловский М.З. Автоматическое управление виброзащитными системами. М.: Наука, 1976. 320 с.

7. Копылов Ю.Р. Динамика процессов виброударного упрочнения: монография. Воронеж: Научная книга, 2011. 568 с.

8. Лонцих П.А., Елисеев С.В. Динамическое качество машин и оборудования как инструмент обеспечения надежности производства и конкурентоспособности процессов. Иркутск: Изд-во Иркутского гос. технического ун-та, 2014. 322 с.

9. Лурье А.И. Операционное исчисление и применение в технических приложениях. М.: Наука, 1959. $368 \mathrm{c}$.

10. Махутов Н.А. Безопасность и риски: системные исследования и разработки. Новосибирск: Наука, 2017. 724 с.

11. Пановко Г.Я. Динамика вибрационных технологических процессов: монография / Ин-т машиноведения им. А.А. Благонравова РАН. М.: Институт компьютерных исследований; Ижевск: Регулярная и хаотическая динамика, 2006. 176 с.

12. De Silva Clarence W. Vibration. Fundamentals and Practice. Boca Raton; London; New York; Washington, D.C., CRC Press, 2000. 957 p.

13. Eliseev S.V., Eliseev A.V. Theory of oscillations. Structural mathematical modeling in problems of dynamics of technical objects. Series Studies in Systems, Decision and Control, Vol. 252. Springer International Publ., Cham, 2020, 521 p.

14. Karnovsky I.A., Lebed E. Theory of Vibration Protection. Springer International Publ., Switzerland, 2016. $708 \mathrm{p}$.

FEFU: SCHOOL of ENGINEERING BULLETIN. 2021. N 3/48

Dynamics, Durability of Machines, Instruments and Equipment www.dvfu.ru/en/vestnikis

DOI: https://doi.org/10.24866/2227-6858/2021-3-3

Eliseev A., Kuznetsov N., Eliseev S.

ANDREY ELISEEV, Candidate of Engineering Sciences, Associate Professor

(Corresponding Author), ResearcherID: N-9357-2016, ORCID: 0000-0003-0222-2507,

ScopusID: 57191957568, eavsh@ya.ru

NIKOLAY KUZNETSOV, Doctor of Engineering Sciences, Professor,

Head of the Department of Design and Standardization in Mechanical Engineering,

ORCID: 0000-0002-3083-0182, knik@istu.edu

Irkutsk National Research Technical University

SERGEY ELISEEV, Doctor of Engineering Sciences, Professor, Advisor to the Rector's

Office for Scientific Work, ORCID: 0000-0001-6876-8786, eliseev_s@inbox.ru

Irkutsk State Transport University

Irkutsk, Russia

System analysis in the assessment of the dynamic states of technical objects: dynamic vibration damping, connectivity of external excitations

Abstract: The scientific and methodological foundations of up-to-date machine dynamics are being developed within the framework of system analysis and methods of structural mathematical modeling. The goal is to develop methods for evaluating, monitoring, and forming dynamic states of objects that operate under vibration loads. The methods of mechanics are used to study mechanical oscillatory systems, which are considered as design schemes of technical objects of technological and transport purposes. Mathematical models are built on the basis of Lagrange equations of the second kind, Laplace integral transformations, and the possibilities of structural mathematical modeling. The technology of constructing mathematical models that allow using the possibilities of implementing dynamic vibration damping modes in systems is developed. A method for estimating the dynamic states in the modes of dynamic vibration damping in systems with two degrees of freedom under the action of two external forces acting together is developed. The results reflect the features of the 
dynamic damping modes, the dependence on the connectivity parameters of external harmonic perturbations. The results of numerical simulations are presented.

Keywords: mechanical oscillatory system, dynamic vibration damping, connectivity of external forces, transfer functions, devices for motion transformation

Contribution of the authors: the authors contributed equally to this article.

The authors declare no conflicts of interests.

\section{REFERENCES}

1. Ganiev R.F., Kononenko V.O. Vibrations of solids: monograph. Moscow, Nauka Publ., 1976, 432 p.

2. Doronin S.V., Shokin Yu. I., Lepikhin A.M., Moskvichev V. V. Modeling of strength and destruction of load-bearing structures of technical systems. Novosibirsk, Nauka Publ., 2005, 249 p.

3. Eliseev A.V., Kuznetsov N.K., Moskovskikh A.O. Dynamics of machines. System representations, structural schemes of communication and elements. Moscow, Innovative Mechanical Engineering, 2019, $381 \mathrm{p}$.

4. Eliseev S.V., Artyunin A.I. Applied theory of oscillations in problems of dynamics of linear mechanical systems. Novosibirsk, Nauka Publ., 2016, 459 p.

5. Yemelyanov S.V., Korovin S.K. New types of feedback: control under uncertainty. Moscow, Nauka Publ., Fizmatlit, 1997, 352 p.

6. Kolovsky M.Z. Automatic control of vibration protection systems. Moscow, Nauka Publ., 1976, $320 \mathrm{p}$.

7. Kopylov Yu.R. Dynamics of vibration-shock hardening processes. Voronezh, Scientific Book Publ., 2011, 568 p.

8. Lontsikh P.A., Eliseev S.V. Dynamic quality of machinery and equipment as a tool for ensuring production reliability and competitiveness of processes. Irkutsk, Publishing House of the Irkutsk State Technical Univ., 2014, 322 p.

9. Lurie A.I. Operational calculus and application in technical applications. Moscow, Nauka Publ., 1959, $368 \mathrm{p}$.

10. Makhutov N.A. Safety and risks: system research and development. Novosibirsk, Nauka Publ., 2017, $724 \mathrm{p}$.

11. Panovko G.Ya. Dynamics of vibration technological processes. Moscow; Izhevsk, Regular and Chaotic Dynamics, 2006, 176 p.

12. De Silva C.W. Vibration. Fundamentals and Practice, monograph. Boca Raton, London, New York, Washington, D.C., CRC Press, 2000, 957 p.

13. Eliseev S.V., Eliseev A.V. Theory of Oscillations. Structural Mathematical Modeling in Problems of Dynamics of Technical Objects. Series: Studies in Systems, Decision and Control, Vol. 252, Springer International Publ., Cham, 2020, 521 p.

14. Karnovsky I.A., Lebed E. Theory of Vibration Protection. Springer International Publ., Switzerland, 2016, 708 p. 